\title{
Detection of RhCE phenotypic variants by serologic routine blood grouping.
}

\author{
Marilène Binsfeld, André Gothot, Mélanie Monfort \\ Immuno-hematology laboratory, Centre Hospitalier Universitaire de Liège, Liège (Belgium)
}

\section{Introduction}

The Rh blood group system is one of the most immunogenic systems, frequently responsible for haemolytic disease of the newborn and transfusion reactions. The $\mathrm{Rh}$ system is also a highly polymorphic system, with numerous phenotypic variants. Among these variants, partial antigens have been described for the RhD antigen (RH:1), but also for common RhCE antigens: RH:2, 3, 4, 5 (C, E, c, e). Carriers of variant $\mathrm{RhCE}$ antigens can develop antibodies against common $\mathrm{Rh}$ proteins, due to the presence of partial antigens and/or the absence of other "public" Rh antigens in these patients (igure 1).

The clinical relevance of such variants becomes appreciable in pregnant women and in chronically transfused patients. In these patients, it is important to identify variant antigens in order to implement preventive transfusion strategies, if possible. For the RhD antigen, reagents used in routine serologic blood grouping reveal an important number of variants. The latter have been extensively studied and classified, and samples showing discrepant serologic results or weak expression levels should be analyzed by molecular biology in order to determine the type of variant $\mathrm{RhD}$. RhCE variants, in contrast, have been less extensively studied and currently, no classification exists. The detection of RhCE variants using routine serologic blood grouping is less common, because reactivity against a given variant antigen can range from 0 to normal agglutination scores, depending on the reagent and the technique that is used. Flegel W.A. (2011), Transfusion and Apheresis Science; Pham B-N et al (2011), Transfusion

\section{Aim}

The aim of this study was the detection of phenotypic RhCE variants in routine patient samples through the comparison of two different serologic blood grouping techniques.

\section{Methods}

Blood grouping samples of 1300 patients (>95\% women, mostly in relation with gynecological follow-up) without recent transfusions have been analyzed using column and microplate agglutination (Figure 2). We defined as "variants" all samples that showed a decreased antigen expression (lower agglutination scores than normal) and/or samples that gave discrepant results between the two techniques (negative versus positive).

\section{Results}

Within the 1300 currently analyzed samples, we detected 20 potential variants: $11 \mathrm{RH} 2(\mathrm{C})$ variants, $1 \mathrm{RH} 3(\mathrm{E})$ variant and $8 \mathrm{RH} 5(\mathrm{e})$ variants. More than $50 \%$ of these patients were of africain ethnic origin. Blood grouping results for these samples are summarized in Table-I. Among the RH2 variants, 7 gave discrepant results in the two techniques. Similar results were observed for one RH5 variant. For most of the other variants, microplate agglutination detected weak expression levels whereas column agglutination gave "normal" positive results. Only for two RH5 variants, opposite results were observed (weak expression detected in column agglutination only), and two RH5 variants showed weak expression in both techniques.

Table-I: Characteristics of serologic blood grouping results for the detected RhCE variants. "DP"=image of double population or weak expression. Agglutination scores for suspected variant antigens are indicated in microplate (/100) and column (/4) agglutination techniques.

\begin{tabular}{|c|c|c|c|c|c|c|}
\hline \multirow{2}{*}{$\begin{array}{l}\text { Variant } \\
\text { antigen }\end{array}$} & \multicolumn{2}{|l|}{ Samples } & \multicolumn{2}{|c|}{ Microplate agglutination } & \multicolumn{2}{|c|}{ Column agglutination } \\
\hline & Observation & Number & Result & Score & Result & Score \\
\hline \multirow{2}{*}{ RH2 (C) } & Discrepant results & 7 & RH: -2,4 (cc) & $<20 / 100(-)$ & RH: 2,4 (Cc) & $4 / 4(+)$ \\
\hline & Weak antigen expression & 4 & RH:2(DP/weak), 4 & $20-85 / 100(\mathrm{DP})$ & $\mathrm{RH}: 2,4$ & $4 / 4(+)$ \\
\hline RH3 (E) & Weak antigen expression & 1 & RH:3(DP/weak), 5 & $<70 / 100(\mathrm{DP})$ & RH:3,5 & $4 / 4(+)$ \\
\hline \multirow[t]{4}{*}{ RH5 (e) } & Discrepant results & 1 & RH:3,-5 (EE) & $<20 / 100(-)$ & $\mathrm{RH}: 3,5$ (Ee) & $3 / 4$ \\
\hline & Weak antigen expression & 3 & RH:5 (DP/weak) & $<90 / 100$ (DP) & RH:5 & $4 / 4(+)$ \\
\hline & & 2 & RH:5 & $>90 / 100(+)$ & RH:5 (DP/weak) & $3 / 4$ \\
\hline & & 2 & RH:5 (DP/weak) & $<80 / 100(\mathrm{DP})$ & RH:5 (DP/weak) & $3 / 4$ or $\mathrm{DP}$ \\
\hline
\end{tabular}

Figure 2: Comparison of RhCE blood grouping results obtained by two different serologic techniques. Examples of normal agglutination patterns for RhCE blood grouping performed by column agglutination (left) and microplate agglutination (right). Results for microplate agglutination are converted into agglutination scores comprised between 0-100, whereas column agglutination is converted into agglutination scores from 0 to $4+$. Both techniques make use of different monoclonal antibodies (different clones) for RhCE typing.

\section{Conclusion}

The frequency of RhCE variants detected within routine samples was $1.5 / 100$. For most (18/20), one of the two techniques gave "normal" agglutination with the suspected variant antigen but for 8 samples, completely discrepant results were obtained by the two techniques.
Thus, only the comparison of results obtained by two different serologic techniques allowed the detection of most variants.Further investigations by molecular biology need to be performed to confirm and identify the types of RhCE variants. 